\title{
Feasibility of diagnosing unstable plaque in patients with acute coronary syndrome using iMap-IVUS*
}

\author{
Jian LIU ${ }^{\dagger}$, Zhao WANG, Wei-min WANG ${ }^{\dagger}$, Qi LI, Yu-liang MA, Chuan-fen LIU, Ming-yu LU, Hong ZHAO \\ (Department of Cardiology, Peking University People's Hospital, Beijing 100044, China) \\ †E-mail: drjianliu@163.com; weiminwang@vip.sina.com \\ Received Aug. 30, 2015; Revision accepted Oct. 26, 2015; Crosschecked Oct. 26, 2015
}

\begin{abstract}
Objective: To compare the plaque composition between stable and unstable plaques, characterize unstable plaque by using iMap-intravascular ultrasound (IVUS), and quantify the diagnostic criteria for unstable plaque. Methods: Thirty-three acute coronary syndrome (ACS) patients who had undergone coronary angiography and IVUS from February 19, 2014 to December 19, 2014 at Peking University People's Hospital were enrolled in the study. Baseline data were collected. The patients were divided into two groups according to their gray-scale IVUS imaging, stable plaque and unstable plaque. A difference-in-difference evaluation was performed using the baseline data and off-line iMap imaging results between the two groups. A receiver operating characteristic (ROC) curve was constructed to obtain the optimal cut-off value to diagnose unstable plaque. Results: Percentages of fibrotic and necrotic tissues, absolute values of lipidic, necrotic, and calcified tissues, and plaque burden were independent predictors for unstable plaque. Absolute necrotic area was the best predictor and exhibited the highest diagnostic value for plaque vulnerability (area under the curve $(A \cup C)=0.806, P=0.000,95 \% \mathrm{Cl}(0.718,0.894)$ ). The cut-off score for predicting unstable plaque was $4.0 \mathrm{~mm}^{2}$. Conclusions: This study attempted to propose a cut-off value based on absolute necrotic area using iMap-IVUS to predict plaque vulnerability in patients with ACS. This score might provide a valuable reference for diagnosing unstable plaque.
\end{abstract}

Key words: Acute coronary syndrome, Intravascular ultrasound, Unstable plaque doi:10.1631/jzus.B1500206

Document code: A

CLC number: R541.4

\section{Introduction}

Sudden ruptures of unstable plaques with a spontaneous thrombosis and consequential interrupted blood flow are characteristics of acute coronary syndrome (ACS) (Fernández-Ortiz et al., 1994). Previous pathological studies showed that most plaques ruptured in ACS are characterized by a thin-capped fibroatheroma (TCFA) with a large necrotic core (Burke et al., 1997). Yamada et al. (2007) demonstrated that plaque vulnerability is associated with several factors such as attenuation plaque,

\footnotetext{
* Corresponding author

* Project supported by the Capital Health Research and Development of Special (No. 2014-2-4085), China

(D) ORCID: Jian LIU, http://orcid.org/0000-0001-9652-0198

(c) Zhejiang University and Springer-Verlag Berlin Heidelberg 2015
}

echolucent or hypoechoic plaque, spotty calcium, thrombosis, and plaque remodeling. Intravascular ultrasound (IVUS) is a widely used standard invasive method to diagnose coronary artery diseases. However, the use of regular IVUS for diagnosis of plaque vulnerability is limited because of at least two reasons: firstly, regular IVUS cannot accurately assess plaque components (Potkin et al., 1990); secondly, to determine vulnerability on gray-scale IVUS images requires well trained physicians with special expertise in the field. The new technology of IVUS, iMapIVUS (Boston Scientific Corp., Fremont, CA, USA), provides a new approach for assessing plaque vulnerability. This system is a spectral analysis of IVUS with a 40-MHz radio frequency system, able to assess the components and tissue characterization in detail such as fibrotic, lipidic, necrotic, and calcified tissues. 
Using such a feature of iMap we should be able to distinguish different tissue components of a plaque and then predict the plaque instability. However, there is no quantitative scale of iMap imaging for diagnosing unstable plaque. Thus, the aims of this study are to establish and quantify the diagnostic criteria of unstable plaque based on iMap images, simplify the diagnostic process, and provide a userfriendly tool for clinicians.

\section{Materials and methods}

\subsection{Study population}

Thirty-three ACS patients underwent coronary angiography (CAG) and iMap-IVUS examination at Peking University People's Hospital (Beijing, China) from February 19, 2014 to December 19, 2014. Exclusion criteria include: contraindications to clopidogrel or aspirin, total occlusion lesion, cardiac shock, and presence of cardiomyopathy, severe anemia, severe renal inadequacy, and malignant tumor.

\subsection{Coronary angiography procedure}

Prior to the CAG procedure, the patients were either administered with $100 \mathrm{mg} / \mathrm{d}$ of aspirin and $75 \mathrm{mg} / \mathrm{d}$ of clopidogrel for at least a week or $300 \mathrm{mg}$ of aspirin and $300 \mathrm{mg}$ of clopidogrel once. CAG was performed after treatment with intravenous unfractionated heparin by using Philips Allura Xper FD10 or Philips Allura Xper FD20 (Philips Healthcare, Best, the Netherlands). CAG was operated based on the American College of Cardiology/American Heart Association guidelines.

\subsection{IVUS imaging and analysis}

All 33 patients received intracoronary administration of 100-200 $\mu \mathrm{g}$ of nitroglycerin before IVUS was conducted. iMap was used with a 40-MHz IVUS catheter (Atlantis SR Pro., Boston Scientific, Natick, MA, USA) to collect data. Imaging results were acquired from beyond the target lesion and rendered with a motorized catheter pullback system set at a speed of $0.5 \mathrm{~mm} / \mathrm{s}$. IVUS data were stored on a hard disk for later offline analysis.

Two independent analysts quantitatively analyzed gray-scale IVUS images based on the criteria of the American College of Cardiology Clinical Expert
Consensus Document on IVUS. Lesions were qualitatively analyzed with QIvus (iMap Basic Viewer 2.1.32.0, Medis medical imaging systems bv, Leiden, the Netherlands). If the plaque was marked by at least one of the following, we catalog the plaque as unstable: (1) plaque rupture, (2) attenuated plaque, (3) echolucent or hypoechoic plaque, (4) microcalcification, (5) thrombosis. Otherwise, the plaque was determined as stable. External elastic membrane (EEM) and lumen cross-sectional area (CSA) were measured using iMap. Plaque burden was calculated as the ratio between plaque CSA (EEM minus lumen CSA) and EEM. iMap-IVUS analysis classified plaques into four color-coded components (green, yellow, red, and blue for fibrotic, lipidic, necrotic, and calcified tissues, respectively). The absolute plaque area and percentage of each tissue component were measured using QIvus. The results in continuous three frames of minimum lumen area (MLA) were recorded. Plaque with acoustic shadowing area and severe calcification or wire artifact was unsuitable for analysis and thus automatically removed.

\subsection{Statistical analysis}

Continuous variables are shown as mean \pm standard deviation (SD), and discrete variables are presented as numbers and percentages. Differences between the group means were assessed by the independent Student's $t$-test. The categorical data were compared using chi-square statistics.

Receiver operating characteristic (ROC) analyses were conducted to evaluate the best predictor of unstable plaque. The maximum Youden index (sensitivity+specificity -1 ) was considered as the optimal cut-off value. A comparative test of multiple correlation detection methods was carried out for multiple thresholds to verify the discriminant effect of the optimal cut-off value (DeLong et al., 1988).

A two-sided $P$-value of $<0.05$ was adopted for statistical significance. The data were analyzed using SPSS 17.0.

\section{Results}

\subsection{Patient characteristics}

Thirty-three patients were enrolled in the study. Among them, 3 patients had ST-segment elevation 
myocardial infarction (STEMI), 3 patients had nonSTEMI, and 27 patients had unstable angina. Table 1 shows the baseline characteristics of the study population.

Table 1 Baseline characteristics of the study population

\begin{tabular}{lc}
\hline \multicolumn{1}{c}{ Characteristics } & Value \\
\hline Sex, $n(\%)$ & $29(87.90)$ \\
$\quad$ Male & $4(12.10)$ \\
Female & $62.94 \pm 10.65$ \\
Age (year) & $25.52 \pm 3.05$ \\
BMI $\left(\mathrm{kg} / \mathrm{m}^{2}\right)$ & $21(63.60)$ \\
Hypertension, $n(\%)$ & $13(39.40)$ \\
DM, $n(\%)$ & $12(36.40)$ \\
Hyperlipidemia, $n(\%)$ & $7(21.20)$ \\
Prior MI, $n(\%)$ & $8(24.20)$ \\
Prior PCI, $n(\%)$ & $12(36.40)$ \\
Smoking, $n(\%)$ & $7(21.20)$ \\
Family history, $n(\%)$ & $3.80 \pm 0.95$ \\
Total cholesterol (mmol/L) & $1.34 \pm 0.58$ \\
Triglyceride (mmol/L) & $2.35 \pm 0.84$ \\
LDL-cholesterol (mmol/L) & $0.92 \pm 0.23$ \\
HDL-cholesterol (mmol/L) & $83.06 \pm 26.44$ \\
Creatinine $(\mu \mathrm{mol} / \mathrm{L})$ & $5.24(4.67,6.23)$ \\
Fast blood glucose (mmol/L) & $0.001(0.001,0.019)$ \\
Troponin I (ng/ml) & \\
\hline BMI: body mass index; DM: diabetes mellitus; MI: myocardial \\
infarction; PCI: percutaneous coronary \\
low-density lipoprotein; HDL: high-density lipoprotein. Data are \\
presented as number (percentage) of patients, mean \pm SD $(n=33)$, \\
or median (interquartile range)
\end{tabular}

Gray-scale IVUS was performed, and 45 lesions were diagnosed from 33 patients. The subjects were divided into stable plaque $(n=12)$ and unstable plaque $(n=33)$ groups according to their gray-scale IVUS imaging. Three continuous frames were analyzed in MLA $(n=135)$. Twenty-six frames $(19 \%)$ were selected as the validation group, and the other 109 frames $(81 \%)$ were the test group. In the test group, 29 and 80 frames were stable and unstable plaques, respectively. Baseline patient characteristics of the two groups are shown in Table 2. The body mass index (BMI) and fasting blood glucose were higher in the unstable plaque group than in the stable plaque group ( $P=0.006$ and $P=0.009$, respectively). Patients with diabetes mellitus (DM), prior myocardial infarction (MI), prior percutaneous coronary intervention (PCI), and family history were higher in the unstable plaques group, whereas total cholesterol and low-density lipoprotein (LDL)-cholesterol were higher in the stable plaque group.

\section{2 iMap-IVUS findings}

The ROC curve was used to detect and identify plaque instability predictors among variables given by iMap, which are summarized in Table 3. It has been reported previously that the absolute lipidic, necrotic, and calcified areas, as well as percentages of fibrotic and necrotic tissues, and plaque burden can be

Table 2 Baseline patient characteristics of the stable plaque and unstable plaque groups

\begin{tabular}{lccc}
\hline \multicolumn{1}{c}{ Characteristics } & Stable plaque $(n=29)$ & Unstable plaque $(n=80)$ & $P$-value \\
\hline Male, $n(\%)$ & $26(89.66)$ & $67(83.75)$ & 0.441 \\
Age $($ year $)$ & $60.93 \pm 9.03$ & $64.28 \pm 10.57$ & 0.133 \\
BMI $\left(\mathrm{kg} / \mathrm{m}^{2}\right)$ & $22.66(22.48,26.57)$ & $24.84(24.23,28.32)$ & 0.006 \\
Hypertension, $n(\%)$ & $17(58.62)$ & $54(67.50)$ & 0.390 \\
DM, $n(\%)$ & $6(20.69)$ & $36(45.00)$ & 0.021 \\
Hyperlipidemia, $n(\%)$ & $8(27.59)$ & $35(43.75)$ & 0.127 \\
Prior MI, $n(\%)$ & $0(0.00)$ & $22(27.50)$ & 0.002 \\
Prior PCI, $n(\%)$ & $2(6.90)$ & $25(31.25)$ & 0.009 \\
Smoking, $n(\%)$ & $14(48.28)$ & $26(32.50)$ & 0.131 \\
Family history, $n(\%)$ & $2(6.90)$ & $19(23.75)$ & 0.049 \\
Total cholesterol $(\mathrm{mmol} / \mathrm{L})$ & $4.30 \pm 0.10$ & $3.65 \pm 0.95$ & 0.020 \\
Triglyceride $(\mathrm{mmol} / \mathrm{L})$ & $1.26(0.92,2.13)$ & $1.38(1.01,1.71)$ & 0.794 \\
LDL-cholesterol $(\mathrm{mmol} / \mathrm{L})$ & $2.83 \pm 0.94$ & $2.19 \pm 0.80$ & 0.001 \\
HDL-cholesterol $(\mathrm{mmol} / \mathrm{L})$ & $0.97(0.75,1.01)$ & $0.99(0.71,1.08)$ & 0.918 \\
Creatinine $(\mu \mathrm{mol} / \mathrm{L})$ & $80.00(72.00,91.00)$ & $75.00(70.00,94.00)$ & 0.802 \\
Fast blood glucose $(\mathrm{mmol} / \mathrm{L})$ & $4.95(4.64,5.26)$ & $5.42(4.69,7.32)$ & 0.009 \\
Troponin ${ }^{*}(\mathrm{ng} / \mathrm{ml})$ & $0.011(0.001,0.031)$ & $0.001(0.001,0.007)$ & 0.031 \\
Statin use, $n(\%)$ & $9(31)$ & $45(56)$ & 0.020 \\
\hline
\end{tabular}

BMI: body mass index; DM: diabetes mellitus; MI: myocardial infarction; PCI: percutaneous coronary intervention; LDL: low-density lipoprotein; HDL: high-density lipoprotein. Data are presented as number (percentage) of patients, mean $\pm \mathrm{SD}$, or median (interquartile range). ${ }^{*}$ Troponin I of $>0.034 \mathrm{ng} / \mathrm{ml}$ means positive 
used to predict plaque instability. The aforementioned parameters were evaluated using multiple detection methods (Tables 4 and 5). We concluded that the absolute necrotic area was the most indicative predictor to detect plaque vulnerability.

Table 3 Measured parameters of iMap-IVUS

\begin{tabular}{lcccc}
\hline Parameter & ROC area & SE & $P$-value & $95 \%$ CI \\
\hline FA $\left(\mathrm{mm}^{2}\right)$ & 0.452 & 0.060 & 0.447 & $(0.334,0.571)$ \\
FP $(\%)$ & 0.747 & 0.056 & 0.000 & $(0.683,0.856)$ \\
LA $\left(\mathrm{mm}^{2}\right)$ & 0.761 & 0.048 & 0.000 & $(0.666,0.855)$ \\
LP $(\%)$ & 0.550 & 0.064 & 0.422 & $(0.426,0.675)$ \\
NA $\left(\mathrm{mm}^{2}\right)$ & 0.806 & 0.045 & 0.000 & $(0.718,0.894)$ \\
NP $(\%)$ & 0.734 & 0.055 & 0.000 & $(0.628,0.841)$ \\
CA $\left(\mathrm{mm}^{2}\right)$ & 0.656 & 0.065 & 0.013 & $(0.529,0.782)$ \\
CP $(\%)$ & 0.538 & 0.065 & 0.546 & $(0.410,0.666)$ \\
PB $(\%)$ & 0.731 & 0.052 & 0.000 & $(0.628,0.834)$ \\
\hline
\end{tabular}

FA: fibrotic area; FP: fibrotic percentage; LA: lipidic area; LP: lipidic percentage; NA: necrotic area; NP: necrotic percentage; CA: calcific area; $\mathrm{CP}$ : calcific percentage; $\mathrm{PB}$ : plaque burden; ROC: receiver operating characteristic; SE: standard error; $\mathrm{CI}$ : confidence interval

Table 4 Comparison test of multiple detection methods 1

\begin{tabular}{|c|c|c|c|c|}
\hline \multirow{2}{*}{ Parameter } & \multicolumn{2}{|c|}{ ROC } & \multirow{2}{*}{ SE } & \multirow{2}{*}{$\begin{array}{c}\text { Asymptotic normal } \\
(95 \% \mathrm{CI})\end{array}$} \\
\hline & Obs & Area & & \\
\hline FP (\%) & 109 & 0.747 & 0.056 & $(0.683,0.856)$ \\
\hline $\mathrm{LA}\left(\mathrm{mm}^{2}\right)$ & 109 & 0.761 & 0.048 & $(0.666,0.855)$ \\
\hline $\mathrm{NA}\left(\mathrm{mm}^{2}\right)$ & 109 & 0.806 & 0.045 & $(0.718,0.894)$ \\
\hline NP (\%) & 109 & 0.734 & 0.055 & $(0.628,0.841)$ \\
\hline $\mathrm{CA}\left(\mathrm{mm}^{2}\right)$ & 109 & 0.656 & 0.065 & $(0.529,0.782)$ \\
\hline PB (\%) & 109 & 0.731 & 0.052 & $(0.628,0.834)$ \\
\hline
\end{tabular}

$H_{0}: \operatorname{area}(\mathrm{FP})=\operatorname{area}(\mathrm{LA})=\operatorname{area}(\mathrm{NA})=\operatorname{area}(\mathrm{NP})=\operatorname{area}(\mathrm{CA})=\operatorname{area}(\mathrm{PB})$. $\mathrm{Chi}^{2}(5)=40.19$, Prob $>\mathrm{Chi}^{2}=0.0000$. FP: fibrotic percentage; LA: lipidic area; NA: necrotic area; NP: necrotic percentage; CA: calcific area; PB: plaque burden; ROC: receiver operating characteristic; Obs: observation sample; SE: standard error; CI: confidence interval

Table 5 Comparison test of multiple detection methods 2 except absolute necrotic area (NA)

\begin{tabular}{lcccc}
\hline \multirow{2}{*}{ Parameter } & \multicolumn{2}{c}{ ROC } & \multirow{2}{*}{ SE } & $\begin{array}{c}\text { Asymptotic normal } \\
(95 \% \mathrm{CI})\end{array}$ \\
\cline { 2 - 3 } & Obs & Area & & $(0.683,0.856)$ \\
FP $(\%)$ & 109 & 0.747 & 0.056 & $(0.666,0.855)$ \\
LA $\left(\mathrm{mm}^{2}\right)$ & 109 & 0.761 & 0.048 & $(0.65)$ \\
NP $(\%)$ & 109 & 0.734 & 0.055 & $(0.628,0.841)$ \\
CA $\left(\mathrm{mm}^{2}\right)$ & 109 & 0.656 & 0.065 & $(0.529,0.782)$ \\
PB $(\%)$ & 109 & 0.731 & 0.052 & $(0.628,0.834)$ \\
\hline
\end{tabular}

$H_{0}: \operatorname{area}(\mathrm{FP})=\operatorname{area}(\mathrm{LA})=\operatorname{area}(\mathrm{NP})=\operatorname{area}(\mathrm{CA})=\operatorname{area}(\mathrm{PB}) . \mathrm{Chi}^{2}(4)=6.01$, Prob $>\mathrm{Chi}^{2}=0.1984$. FP: fibrotic percentage; LA: lipidic area; NP: necrotic percentage; $\mathrm{CA}$ : calcific area; PB: plaque burden; ROC: receiver operating characteristic; Obs: observation sample; SE: standard error; CI: confidence interval
Youden index (sensitivity+specificity-1) maximum value was selected as the optimal cut-off value, and the optimal value was $4.0 \mathrm{~mm}^{2}$. When the absolute necrotic area was larger than $4.0 \mathrm{~mm}^{2}$, the plaque was diagnosed as unstable plaque. Based on previous reports, it became apparent that the absolute necrotic area was the most relevant predictor to indicate plaque instability (Fig. 1).

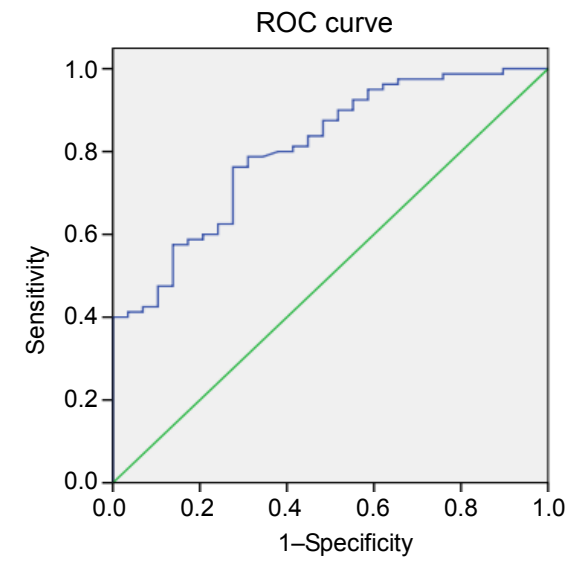

Fig. 1 ROC curve of absolute necrotic area in predicting unstable plaque

$\mathrm{AUC}=0.806, P=0.000,95 \%$ CI $(0.718,0.894)$

\subsection{Verification}

The cut-off values were divided into test, verification, and whole groups for verification (Table 6).

Table 6 Verification of absolute necrotic area larger than $4.0 \mathrm{~mm}^{2}$ in predicting unstable plaque

\begin{tabular}{lcccc}
\hline \multicolumn{1}{c}{ Group } & $\begin{array}{c}\text { Sensitivity } \\
(\%)\end{array}$ & $\begin{array}{c}\text { Specificity } \\
(\%)\end{array}$ & $\begin{array}{c}\text { Positive } \\
(\%)\end{array}$ & $\begin{array}{c}\text { Negative } \\
(\%)\end{array}$ \\
\hline Test & 78.75 & 65.52 & 86.30 & 52.78 \\
Verification & 78.95 & 57.14 & 83.33 & 50.00 \\
Total & 78.75 & 63.89 & 86.30 & 56.10 \\
\hline
\end{tabular}

\section{Discussion}

As is well known, the unstable plaque, also called vulnerable plaque, plays an important role in ACS. With the development of endovascular techniques, the positive detection rate of unstable plaque has greatly improved. IVUS is currently considered as a promising technique used to detect plaque vulnerability (König and Klauss, 2007). Gray-scale IVUS images not only reveal the changes of the vessel lumen but also show the vascular wall structure. 
Previous studies confirmed that thin fibrous cap, large lipid core, plaque rupture, thrombosis, and positive remodeling are related to plaque vulnerability and instability. Accurate diagnosis of plaque vulnerability requires expertise because distinguishing lipid and fiber, plaque hemorrhage and thrombosis on grayscale IVUS images is difficult. Thus, inexperienced doctors have difficulty in identifying vulnerable plaque. Applications of virtual histology IVUS (VH-IVUS; iMap) and integrated backscatter (IB)IVUS have recently emphasized IVUS values. VHIVUS considers TCFA as vulnerable plaque when there are at least three consecutive frame plaque burdens $>40 \%$ and necrotic core $>10 \%$ (Stone et al., 2011). iMap is a new software package used to assess plaque composition by using a backscattered ultrasound frequency spectrum, sampled with a $40-\mathrm{MHz}$ IVUS catheter. Given that VH-IVUS and iMap are two different IVUS-based tissue characterization systems, the VH criterion may not be directly applicable in the interpretation of iMap data (Sathyanarayana et al., 2009; Shin et al., 2011).

The results in the present study showed that the BMI was higher in unstable plaque than in stable plaque, as well as the incidence rates of DM, old MI, past PCI, and coronary heart disease family history. Meanwhile, laboratory examination results suggested that fasting blood glucose was higher in unstable plaque than in the stable plaque, which is consistent with previous studies (Araki et al., 2013). Nasu et al. (2008) evaluated the characteristics of atheromatous plaque in 90 patients with VH-IVUS. Compared with non-diabetic patients, the proportion of necrosis core $(11.0 \%$ vs. $7.6 \%, P=0.03)$ and dense calcified tissue $(5.6 \%$ vs. $2.9 \%, P=0.01)$ in DM patients significantly increased. Despres et al. (2001) showed that the high BMI, high rate in coronary heart disease, and high death risk from coronary disease are related to obesity, lipid, and glucose metabolism.

Yla-Herttuala et al. (1994) have also demonstrated that cholesterol, especially LDL-cholesterol, has a vital role in atherosclerotic plaque formation and plaque vulnerability. However, in the present study, total cholesterol $((3.65 \pm 0.95) \mathrm{mmol} / \mathrm{L}$ vs. $(4.30 \pm 0.10) \mathrm{mmol} / \mathrm{L}, P=0.02)$ and LDL-cholesterol $((2.19 \pm 0.80) \mathrm{mmol} / \mathrm{L}$ vs. $(2.83 \pm 0.94) \mathrm{mmol} / \mathrm{L}, P=0.001)$ were lower in unstable plaque than in stable plaque, which was inconsistent with the former studies. This result is explained by the usage discrepancy of statin. The percentage of statin use was significantly greater in the unstable plaque group than in the stable plaque group ( $56 \%$ vs. $31 \%, P=0.020$ ).

In the current study, the absolute lipidic, necrotic, and calcified areas, percentages of fibrotic and necrotic tissues, and plaque burden could predict plaque vulnerability and exhibit better diagnostic value and accuracy. Previous iMap studies showed that high percentages of lipidic, necrotic, and calcified tissues and large plaque burdens seem related to plaque vulnerability (Araki et al., 2013). Kozuki et al. (2013) evaluated 93 culprit lesions from 87 patients by using iMap. The feasibility of iMap technology is confirmed by comparing plaque characteristics in ACS and non-ACS patients. Furthermore, ACS patients exhibited larger lipid and necrotic areas than non-ACS patients. Multivariate analysis showed that the lipidic region was the independent factor $(\mathrm{OR}=1.5$, $P=0.04)$. Similarly, Trusinskis et al. (2013) analyzed 63 STEMI patients with culprit and non-culprit arteries using iMap. A higher percentage of necrotic tissue was found in the culprit lesion than in the non-culprit lesion. However, a comparatively high percentage of necrotic tissue was observed in culprit lesions proximal to the non-culprit from the index procedure by iMap IVUS $((31.9 \pm 10.0) \%$ vs. $(27.8 \pm 11.8) \%, P=0.012)$. Araki et al. (2013) used iMap in 174 vessels from 146 stable angina pectoris patients. The patients were divided into two groups based on a remodeling index: positive remodeling group (PR; remodeling index $>1.0$ ) and a non-positive remodeling group (non-PR; remodeling index $\leq 1.0$ ). The results showed that more attenuation plaques were observed in the PR group than in the non-PR group ( $40.8 \%$ vs. $12.1 \%, P<0.0001)$. In the PR group, the percentages of lipidic and necrotic areas at the MLA sites were greater $(7.22 \%$ vs. $6.03 \%, P<0.05$ and $22.08 \%$ vs. $14.71 \%, P<0.001$, respectively), whereas that of the fibrotic area was smaller $(54.82 \%$ vs. $61.42 \%, P<0.05)$.

The present study revealed that the absolute necrotic area was the most indicative predictor for predicting plaque vulnerability, and the optimal cut-off value was $4.0 \mathrm{~mm}^{2}$. The cut-off value was verified and showed that the sensitivity and specificity were high. The optimal cut-off value had a high positive predictive value, which suggested that intensive 
therapy was needed when the plaque absolute necrosis area $>4 \mathrm{~mm}^{2}$. However, the negative predictive value was low. The possible reasons include the following: the absolute necrosis area is associated with vessel and plaque areas, and the sample size of this study is small, and thus more cases are required to confirm these findings.

\section{Limitations}

This study has several limitations. Firstly, it is a single-center retrospective analysis with a small sample size; thus, selection bias cannot be completely avoided. The follow-up time was short. Secondly, the average follow-up time with the 33 patients was $(254.7 \pm 87.4) \mathrm{d}$. Only three patients had recurring angina pectoris, and no major adverse cardiovascular events (MACEs; i.e. cardiac death, nonfatal myocardial infarction, or target lesion revascularization) occurred. Given the short follow-up time, the number of incidences of clinical event was low; thus, the correlation between the unstable plaque and the clinical events could not be analyzed. The follow-up duration should be extended and provide more information for the MACE rate. Thirdly, several plaque areas were unsuitable for analysis because of calcification and guide-wire artifacts. Finally, analyzed lesions constituted only a small portion of the entire coronary arterial system; not all patients had undergone PCI.

\section{Conclusions}

This study attempted to propose a cut-off value based on absolute necrotic area using iMap-IVUS to predict plaque vulnerability in patients with ACS. This score might provide a valuable reference for diagnosing unstable plaque.

\section{Compliance with ethics guidelines}

Jian LIU, Zhao WANG, Wei-min WANG, Qi LI, Yu-liang MA, Chuan-fen LIU, Ming-yu LU, and Hong ZHAO declare that they have no conflict of interest.

All procedures followed were in accordance with the ethical standards of the responsible committee on human experimentation (institutional and national) and with the Helsinki Declaration of 1975, as revised in 2008 (5). Informed consent was obtained from all patients for being included in the study.

\section{References}

Araki, T., Nakamura, M., Utsunomiya, M., et al., 2013. Visualization of coronary plaque in arterial remodeling using a new $40-\mathrm{MHz}$ intravascular ultrasound imaging system. Catheter. Cardiovasc. Interv., 81(3):471-480. [doi:10. 1002/ccd.23501]

Burke, A.P., Farb, A., Malcom, G., et al., 1997. Coronary risk factors and plaque morphology in men with coronary disease who died suddenly. N. Engl. J. Med., 336(18): 1276-1282. [doi:10.1056/NEJM199705013361802]

DeLong, E.R., DeLong, D.M., Clarke-Pearson, D.L., 1988. Comparing the areas under two or more correlated receiver operating characteristic curves: a nonparametric approach. Biometrics, 44(3):837-845. [doi:10.2307/253 1595]

Despres, J.P., Lemieux, I., Prud'homme, D., 2001. Treatment of obesity: need to focus on high risk abdominally obese patients. $B M J, \mathbf{3 2 2}$ (7288):716-720.

Fernández-Ortiz, A., Badimon, J.J., Falk, E., et al., 1994. Characterization of the relative thrombogenicity of atherosclerotic plaque components: implications for consequences of plaque rupture. J. Am. Coll. Cardiol., 23(7): 1562-1569. [doi:10.1016/0735-1097(94)90657-2]

König, A., Klauss, V., 2007. Virtual histology. Heart, 93(8): 977-982. [doi:10.1136/hrt.2007.116384]

Kozuki, A., Shinke, T., Otake, H., et al., 2013. Feasibility of a novel radiofrequency signal analysis for in-vivo plaque characterization in humans: comparison of plaque components between patients with and without acute coronary syndrome. Int. J. Cardiol., 167(4):1591-1596. [doi:10. 1016/j.ijcard.2012.04.102]

Nasu, K., Tsuchikane, E., Katoh, O., et al., 2008. Plaque characterization by Virtual Histology intravascular ultrasound analysis in type II diabetic patients. Heart, 94(4): 429-433.

Potkin, B.N., Bartorelli, A.L., Gessert, J.M., et al., 1990. Coronary artery imaging with intravascular high-frequency ultrasound. Circulation, 81(5):1575-1585. [doi:10.1161/ 01.CIR.81.5.1575]

Sathyanarayana, S., Carlier, S., Li, W., et al., 2009. Characterisation of atherosclerotic plaque by spectral similarity of radiofrequency intravascular ultrasound signals. EuroIntervention, 5(1):133-139. [doi:10.4244/EIJV5I1A21]

Shin, E.S., Garcia-Garcia, H.M., Ligthart, J.M., et al., 2011. In vivo findings of tissue characteristics using iMap ${ }^{\mathrm{TM}}$ IVUS and Virtual Histology ${ }^{\mathrm{TM}}$ IVUS. EuroIntervention, 6(8):1017-1019. [doi:10.4244/EIJV618A175]

Stone, G.W., Maehara, A., Lansky, A.J., et al., 2011. A prospective natural-history study of coronary atherosclerosis. N. Engl. J. Med., 364(3):226-235. [doi:10.1056/NEJMoa 1002358]

Trusinskis, K., Juhnevica, D., Strenge, K., et al., 2013. iMap intravascular ultrasound evaluation of culprit and non-culprit lesions in patients with ST-elevation myocardial infarction. Cardiovasc. Revasc. Med., 14(2):71-75. 
[doi:10.1016/j.carrev.2012.12.001]

Yamada, R., Okura, H., Kume, T., et al., 2007. Histological characteristics of plaque with ultrasonic attenuation: a comparison between intravascular ultrasound and histology. J. Cardiol., 50(4):223-228.

Yla-Herttuala, S., Palinski, W., Butler, S.W., et al., 1994. Rabbit and human atherosclerotic lesions contain IgG that recognizes epitopes of oxidized LDL. Arterioscler. Thromb. Vasc. Biol., 14(1):32-40. [doi:10.1161/01.ATV. 14.1.32]

\section{中文概要}

题 目: 关于 iMap-IVUS 指导急性冠脉综合征患者不稳 定斑块诊断价值的研究

目 的: 比较稳定斑块与不稳定斑块虚拟组织学血管内超
声（iMap-IVUS）评价斑块组成成分的不同特点, 量化不稳定斑块的诊断标准。

创新点: 首次提出运用 iMap-IVUS 评价不稳定斑块的量化 标准, 简化不稳定斑块的诊断。

方 法: 连续入选接受冠状动脉造影及血管内超声检查的 急性冠脉综合征患者, 收集包括人口学资料、既 往史、实验室生化检查、冠脉病变情况等在内的 基线数据。分析所有患者的灰阶 IVUS 影像, 根 据影像学特点, 将斑块分为稳定斑块和不稳定斑 块。比较两组患者的基线资料, 并离线对比分析 两组 iMap-IVUS 影像结果, 应用受试者工作特征 曲线 (ROC) 获取诊断不稳定斑块的最佳國值并 进行验证。

结 论: 研究运用 iMap-IVUS 提出急性冠脉综合患者不稳 定斑块的诊断阈值, 对不稳定斑块的诊断有一定 价值。

关键词：急性冠脉综合征; 血管内超声; 不稳定斑块 